\title{
ON THE ANOMALOUS SINGULARITIES OF THE SOLUTIONS TO SOME CLASSES OF WEAKLY HYPERBOLIC SEMILINEAR SYSTEMS. EXAMPLES
}

\author{
PETAR POPIVANOV \\ Institute of Mathematics and Informatics \\ Bulgarian Academy of Sciences \\ 1113 Sofia, Bulgaria \\ E-mail: popivano@math.bas.bg \\ IORDAN IORDANOV \\ Faculty of Mathematics and Informatics \\ Sofia University "St. Kl. Ohridski" \\ blvd. "J. Bourchier" N. 5, Sofia, Bulgaria
}

\begin{abstract}
This paper deals with the newly observed singularities of the solutions of some specific examples of weakly hyperbolic semilinear systems in $\mathbf{R}^{2}$. Two, respectively three, characteristics are supposed to be mutually tangential at the origin only and the initial data are continuous only. The exact strength of the new-born singularities is investigated too.
\end{abstract}

1. Introduction. When studying the propagation of singularities of the solutions to semilinear hyperbolic and non-strictly hyperbolic equations and systems, interesting new effects in comparison with the linear case can appear. The interaction of the singularities propagating along several characteristics crossing at some point (surface) could give rise of new singularities propagating along the outgoing characteristics starting from that point (surface). In many cases the new-born singularities are weaker than the initial ones. We shall mention only the papers of Beals [1], Bony [2], [3], Chemin [4], Hörmander [6], Melrose-Ritter [9] (cf. also Gramchev [5] for appearance of Gevrey ultradistributional singularities). The propagation of jump type discontinuities for hyperbolic and weakly hyperbolic systems in $\mathbf{R}^{2}$ was considered by Rauch-Reed [11], [12], John [8], Micheli [10],

Research of the first author partially supported by the joint research project on nonlinear hyperbolic equations between Bulgarian Academy of Sciences and CNR (Italy).

2000 Mathematics Subject Classification: Primary 35L60, 35L67.

Key words and phrases: weakly hyperbolic system, anomalous singularity.

The paper is in final form and no version of it will be published elsewhere. 
Iordanov [7] and others. We specify here the main results from [10], [7] in the case of continuous Cauchy data and we prove that the strength of the newly observed singularity propagating along a transversal outgoing characteristic is rather different from the strength of that singularity in the case of sufficiently smooth Cauchy data. We are dealing with jump discontinuities in all our investigations. Without loss of generality we assume that 2 or 3 characteristics are tangential to each other of finite order at the origin.

\section{Formulation and proof of the main results}

2.1. Consider the weakly hyperbolic system in the plane $\mathbf{R}^{2}$ :

$$
\left\{\begin{array}{l}
X u=\left(\partial_{t}+p t^{p-1} \partial_{x}\right) u=0 \\
\partial_{t} v=u \\
D z=\left(\partial_{t}+\partial_{x}\right) z=u v
\end{array}\right.
$$

where $p>0$ is an even integer and the initial data $u_{0}(x), v_{0}(x)=z_{0}(x)=0$ are prescribed for $t=-T<0$.

Assume first that $u_{0}(x)=c_{1}\left(x-x_{0}\right)^{k+1}, k \geq 1, k$ - integer, $x \leq x_{0} ; u_{0}(x)=$ $c_{2}\left(x-x_{0}\right)^{k+1}, x>x_{0}, c_{1} c_{2}>0, c_{1} \neq c_{2}$. Obviously, $u_{0} \in C^{k}\left(\mathbf{R}^{1}\right)$ and $\partial_{x}^{k+1} u$ has a jump discontinuity at $x_{0}$. We shall say then that $u_{0}$ has a $k$-order finite jump. The characteristics of the vector fields $X$ and $\frac{\partial}{\partial t}$ are tangential each to other at 0 and $D$ is transversal with respect to them. L. Micheli has proved in [10] that a new-born singularity of the component $z$ appears at the origin and propagates along the characteristic $x-t=0$, $t>0$. Moreover, $z \in C^{(2 k+3) p}$ near that outgoing characteristic and it has jump type discontinuities of order $(2 k+3) p$, i.e. some derivative of $z$ of order $(2 k+3) p+1$ in transversal direction to $D$ possesses jump along the characteristic mentioned above. Certainly, $z \in C^{\infty}$ in a neighbourhood of $x-t=0, t<0$. We shall see that the picture is quite different if $k=0$.

Denote by $C_{1}, C_{2}, C_{3}$ the characteristics of $X, \partial_{t}, D$ passing through the origin (see Fig. 1). The characteristics of the same vector fields passing through some point $(\bar{x}, \bar{t})$ are given respectively by: $x=t^{p}+\bar{x}-\bar{t}^{p}, x=\bar{x}, x=t+\bar{x}-\bar{t}$. Put $x_{0}=T^{p}$. Then

$$
u(\bar{x}, \bar{t}) \equiv u\left(T^{p}+\bar{x}-\bar{t}^{p},-T\right)=u_{0}\left(x_{0}+\bar{x}-\bar{t}^{p}\right) .
$$

Consider now the following cases of Cauchy data:
a) $u_{0}(x)= \begin{cases}x-x_{0}, & x \leq x_{0} \\ 2\left(x-x_{0}\right), & x>x_{0}\end{cases}$
b) $u_{0}(x)= \begin{cases}x-x_{0}, & x \leq x_{0} \\ 0, & x>x_{0}\end{cases}$
c) $u_{0}(x)= \begin{cases}0, & x \leq x_{0} \\ x-x_{0}, & x>x_{0}\end{cases}$
d) $u_{0}(x)= \begin{cases}1, & x \leq x_{0} \\ 1+x-x_{0}, & x>x_{0}\end{cases}$
e) $u_{0}(x)= \begin{cases}1+x-x_{0}, & x \leq x_{0} \\ 1, & x>x_{0}\end{cases}$

There are no difficulties to give explicit formulas for the component $u$ of (1) in the cases a)-e) by applying (2). For example, in case a) $u=x-t^{p}$ for $x \leq t^{p}, u=2\left(x-t^{p}\right)$, $x \geq t^{p}$; in case b) $u=x-t^{p}$ for $x \leq t^{p}, u=0$ if $x \geq t^{p}$; in case c) $u=0$ for $x \leq t^{p}$, $u=x-t^{p}$ if $x \geq t^{p}$ etc. 


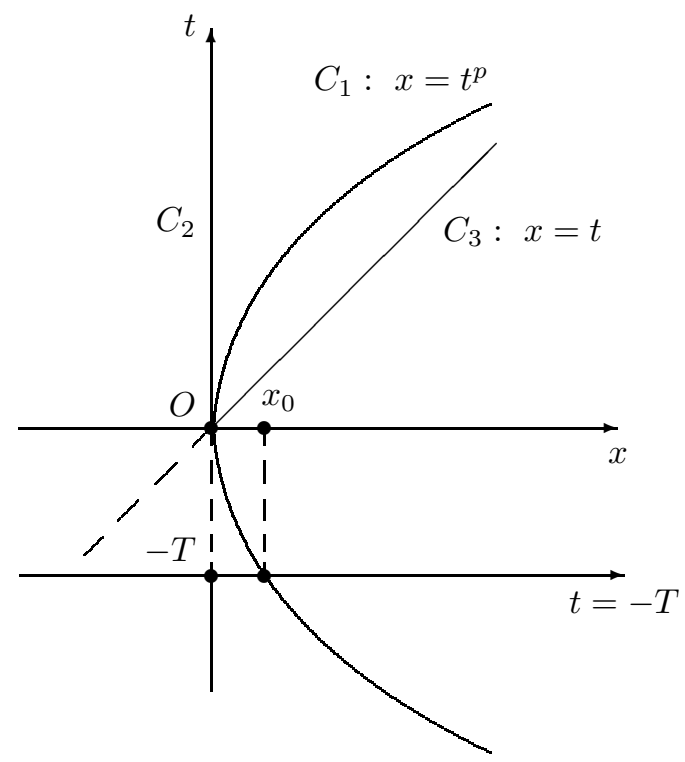

Fig. 1

Having in mind that $v(x, t)=\int_{-T}^{t} u(x, \tau) d \tau$ we introduce the functions:

$$
\begin{gathered}
A(x, t)=\int_{-T}^{t}\left(x-\tau^{p}\right) d \tau, \\
B(x, t)=\int_{-x^{1 / p}}^{t} u(x, \tau) d \tau, x>0, \\
C(x)=\int_{-x^{1 / p}}^{x^{1 / p}}\left(x-\tau^{p}\right) d \tau=\frac{2 p}{p+1} x^{1+1 / p}, x>0 .
\end{gathered}
$$

It is rather easy to compute the values of $v$ in the following three subdomains of $\mathbf{R}^{2}$ : $\{x<0\} \cup\left\{0<x<t^{p}, t<0\right\},\left\{t^{p}<x\right\}$ and the curvilinear angle between the characteristics $C_{1}^{+}, C_{2}^{+}, C_{1}^{+}=C_{1} \cap\{x>0\}, C_{2}^{+}=C_{2} \cap\{x>0\}$ (see Fig. 1). We shall denote these values by $v_{1}, v_{2}, v_{3}$. Then in case a) $v_{1}=A, v_{2}=A+B, v_{3}=A+C$; in case b) $v_{1}=A, v_{2}=A-B, v_{3}=A-C(x)$, in case c) $v_{1}=0, v_{2}=B, v_{3}=C(x)$ etc.

Suppose that $(x, t) \in C_{3}, x>0$ and consider the $D$-characteristics $C_{3}^{ \pm}$passing through the points $\left(x^{+}, t^{+}\right),\left(x^{-}, t^{-}\right)$located near $(x, t)$ above and, respectively, under the characteristic $C_{3}$, i.e. $C_{3}^{+}: x-t=\sigma^{+}=x^{+}-t^{+}<0, C_{3}^{-}: x-t=\sigma^{-}=x^{-}-t^{-}>0$. The symbols $a_{1}, b_{1}$ stand for the ordinates of the crossing points of $C_{3}^{ \pm}$with the ordinate axes while $a_{2}, b_{2}$ stand for the ordinates of the crossing points of $C_{3}^{ \pm}$with $C_{1}$. Then $a_{1}+x-t=0, b_{1}+x-t=0$ and $a_{2}(\sigma), \sigma<0, b_{2}(\sigma), \sigma>0$ satisfy in a neighbourhood of $\sigma=0$ the equation

$$
c^{p}(\sigma)=c(\sigma)+\sigma, \quad c(0)=0 .
$$

According to the implicit function theorem $a_{2}, b_{2} \in C^{\infty}, c(\sigma)=-\sigma+\sigma^{p}+O\left(\sigma^{p+1}\right)$, $\sigma \rightarrow 0, \sigma=\sigma^{ \pm}$.

Our next step is to compute in a small neighbourhood of $\left(x^{+}, t^{+}\right)$(or $\left.\left(x^{-}, t^{-}\right)\right)$the corresponding values $z^{+}\left(z^{-}\right)$of $z(x, t)$ and to find out the limit of $z^{+}\left(z^{-}\right)$and its 
derivatives up to some order in some direction transversal to $C_{3}$ for $\sigma \rightarrow 0^{+}\left(\sigma \rightarrow 0^{-}\right)$. Thus, we have in case a):

$$
\begin{gathered}
z^{+}(x, t)=\int_{-T}^{a_{1}} A u d \tau+\int_{a_{1}}^{a_{2}}(A+C) u d \tau+\int_{a_{2}}^{t}(A+B) u d \tau \\
z^{-}(x, t)=\int_{-T}^{b_{1}} A u d \tau+\int_{b_{1}}^{b_{2}} A u d \tau+\int_{b_{2}}^{t}(A+B) d \tau
\end{gathered}
$$

and we are integrating in both cases along the straight line $(\tau+x-t, \tau)$, i.e. $\left(\tau+\sigma^{ \pm}, \tau\right)$.

One can guess that jump discontinuities can appear from the "asymmetric" term $\int_{a_{1}}^{a_{2}} C(\tau+x-t) u(\tau+x-t, \tau) d \tau$ only, i.e. from the term with underintegral function participating in $z^{+}$but not in $z^{-}$. The standard change $\tau+x-t=s^{p}$ in the last integral shows that

$$
\Gamma=\int_{a_{1}}^{a_{2}} C u d \tau=p \int_{0}^{a_{2}} \frac{2 p}{p+1} s^{p+1}\left[s^{p}-\left(s^{p}-\sigma\right)^{p}\right] s^{p-1} d s,
$$

as $a_{2}+\sigma=a_{2}^{p}, a_{1}+\sigma=0, \sigma<0$.

The terms having minimal powers in $(s, \sigma)$ and participating in the polynomial under integral (5) are $s^{3 p}$ and $s^{2 p} \sigma^{p}$. Thus, the leading term in (5) near $\sigma^{+}=x-t \rightarrow 0^{-}$ is const. $\left(\frac{a_{2}^{3 p+1}}{3 p+1}-\frac{a_{2}^{2 p+1}}{2 p+1} \sigma_{+}^{p}\right)$. The same change shows that $\int_{a_{2}}^{t} B u d \tau \in C^{\infty}(x>0)$ etc. In this way we conclude that each derivative of the asymmetric term $\Gamma$ of order not exceeding $3 p$ tends to 0 for $\sigma^{+} \rightarrow 0$. On the other hand, the leading term in $\Gamma$ is given by

$$
\frac{p\left(\sigma^{+}\right)^{3 p+1}}{(2 p+1)(3 p+1)}\left(1+O\left(\sigma^{+}\right)\right), \quad \sigma^{+} \rightarrow 0 .
$$

Consequently, $z \in C^{3 p}$ in a neighbourhood of $C_{3}$ contained inside the parabola $C_{1}$, while some transversal with respect to $C_{3}$ derivative of $z$ of order $3 p+1$ has a jump discontinuity at $C_{3}$. The same result holds in case b).

In case c) $z^{+}=\int_{a_{2}}^{t} B u d \tau, z^{-}=\int_{b_{2}}^{t} B u d \tau$ and therefore $z \in C^{\infty}$ in a neighbourhood of $C_{3}$ located inside $C_{1}$.

In case d) jump discontinuities can appear by the following term only (leading term):

$$
\int_{a_{1}}^{a_{2}} \frac{2 p}{p+1}(\tau+x-t)^{(p+1) / p} d \tau=\frac{2 p^{2}}{(p+1)(2 p+1)} a_{2}^{2 p+1} .
$$

Thus, $z \in C^{2 p}$ near $C_{3}$ and the transversal derivatives of $z: \partial_{x}^{2 p+1} z, \partial_{t}^{2 p+1}$ have finite jumps along $C_{3}$ inside $C_{1}$.

In case e) the component $z$ has finite jump discontinuity of order $2 p$. So we come to the following proposition.

Proposition 1. Consider the weakly hyperbolic semilinear system (1) with Cauchy data $u_{0}$ having finite jump of order $k=0$ at some point $x_{0}$ and $v_{0} \equiv z_{0} \equiv 0$. Then in general the component $z$ does not have jump of exact order $(2 k+3) p$ along the characteristic $C_{3}$ located inside the parabola $C_{1}$.

The examples a), b) correspond to the central results in Micheli [10], Iordanov [7], claiming that the newly created singularity of the component $z(x, t)$ has a finite jump discontinuity of order $(2 k+3) p, k=0$ along the part of $C_{3}$ located inside $C_{1}$. On the 
other hand, examples c), d), e) contradict the main results in the same papers as either $z \in C^{\infty}$ near $C_{3}$ or $z$ possesses a finite jump discontinuity along $C_{3}$ and inside $C_{1}$ of order $(k+2) p<(2 k+3) p, k=0$. Certainly, this is a new effect.

2.2. Consider now the weakly hyperbolic semilinear system

(6)

$$
\left\{\begin{aligned}
X u= & \left(\partial_{t}+p t^{p-1} \partial_{x}\right) u=0 \\
\partial_{t} v= & u \\
Y w= & \left(\partial_{t}-p t^{p-1} \partial_{x}\right) w=u+v \\
D z= & \left(\partial_{t}+\partial_{x}\right) z=\alpha u v+\beta u w+\gamma v w, \\
& \alpha, \beta, \gamma=\text { const., } p-\text { even integer } \geq 2
\end{aligned}\right.
$$

with Cauchy data $\left.u\right|_{t=-T}=u_{0}(x), T>0, v_{0}=w_{0}=z_{0}=0$. The constants $\alpha, \beta, \gamma$ are assumed to be different from 0 .

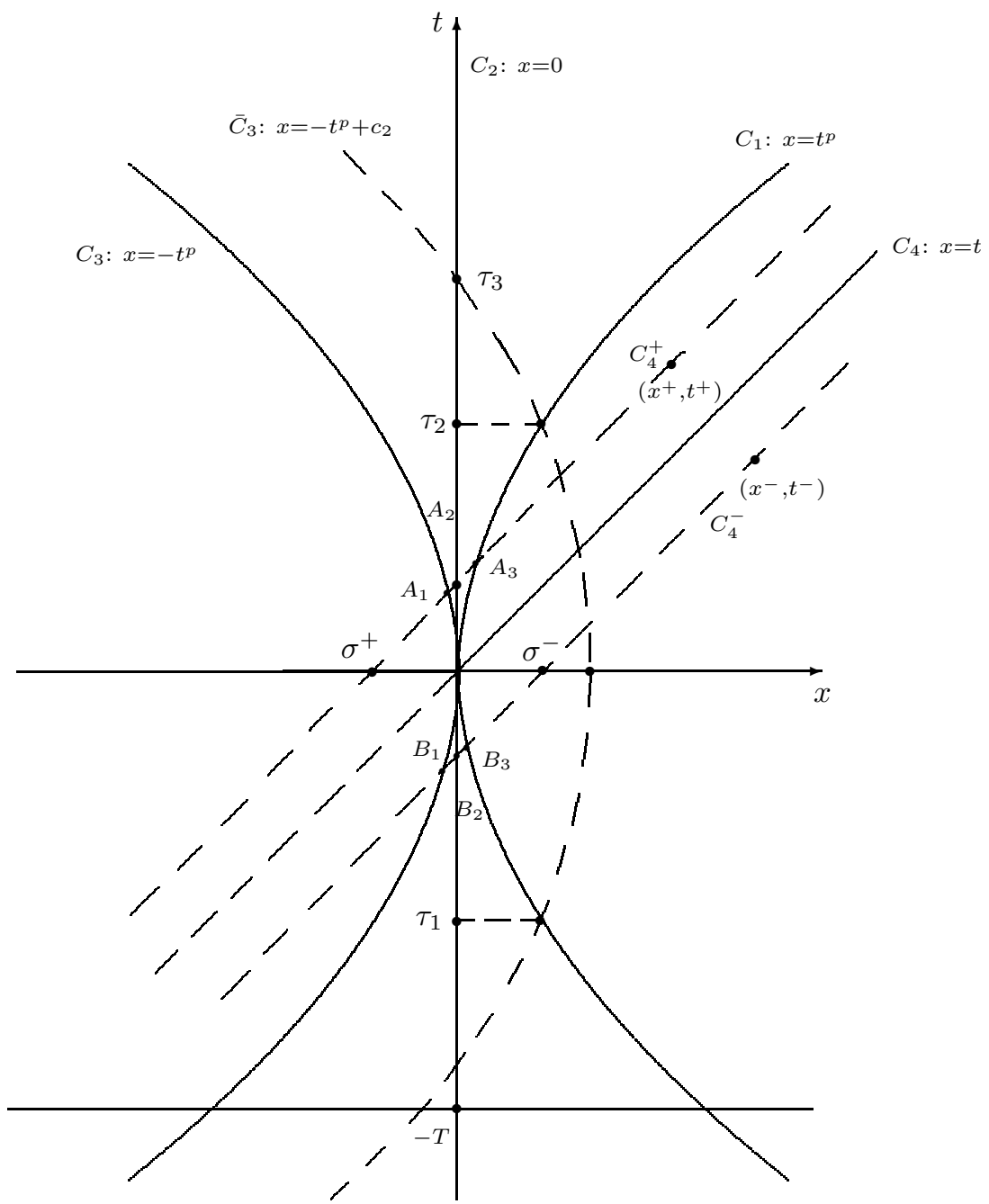

Fig. 2 
The characteristics of the vector fields $X, \partial_{t}, Y, D$ passing through the point $(\bar{x}, \bar{t})$ are respectively: $x=t^{p}+c_{1}, c_{1}=\bar{x}-\bar{t}^{p}, x=\bar{x}, x=-t^{p}+c_{2}, c_{2}=\bar{x}+\bar{t}^{p}, x-t=\bar{x}-\bar{t}$. Put $C_{1}: x=t^{p}, C_{2}: x=0, C_{3}: x=-t^{p}, \bar{C}_{3}: x=-t^{p}+c_{2}, C_{4}: x=t$. Obviously, $C_{1}, C_{2}, C_{3}$ are tangential each to other characteristics at 0 and $C_{4}$ is transversal to them at 0 . The initial function $u_{0}$ is defined by $\mathrm{d}$ ) from (1) (see Fig. 2). Then $u=u_{1}=1$ outside the parabola $C_{1}$ and $u=u_{2}=1+x-t^{p}$ inside it. The values of $v$ in the three subdomains introduced above are:

$$
\begin{aligned}
v_{1}=t+T, \quad v_{2} & =v_{1}+x t-\frac{t^{p+1}}{p+1}+\frac{p}{p+1} x^{1+1 / p}, \quad x>0, \\
v_{3} & =v_{1}+\frac{2 p}{p+1} x^{1+1 / p}, \quad x>0 .
\end{aligned}
$$

TECHNICAL REMARK. When estimating the strength of the new-born singularities along $C_{3} \cap\{x>0\}$ we need some additional calculations. Thus consider the function $\theta(s)=s /\left(s^{p}-\sigma\right), s \geq 0$, where $\sigma<0,|\sigma| \ll 1, a_{3}=-\sigma+\sigma^{p}+O\left(\sigma^{p+1}\right), a_{3}^{p}=a_{3}+\sigma$. Then $\theta(0)=0$ and $\theta$ is monotonically increasing on the interval $\left[0, s_{0}\right)$ and is monotonically decreasing for $s \geq s_{0}$, where $s_{0}=(\sigma /(1-p))^{1 / p}>0$. On the other hand, $0 \leq a_{3} \leq s_{0}(\sigma)$. Therefore, $0 \leq s \leq a_{3} \Rightarrow 0 \leq \theta(s) \leq \theta\left(a_{3}\right)=1$. There are no difficulties to see that $\int_{0}^{a_{3}}\left(s^{p}-\sigma\right)^{p+1} s^{p-1} d s=-\sigma^{p+1} a_{3}^{p} / p+O\left(\sigma^{3 p}\right), \sigma \rightarrow 0, \sigma<0$.

Let $c_{2}=x+t^{p}$. Then $w(x, t)=\int_{-T}^{t}(u+v)\left(c_{2}-\tau^{p}, \tau\right) d \tau$. The crossing points of $\bar{C}_{3}$ with $C_{1}$ and $C_{2}^{+}$have the following ordinates: $\tau_{1}=-\left(c_{2} / 2\right)^{1 / p}, \tau_{2}=-\tau_{1}, \tau_{3}=c_{2}^{1 / p}$. Define now the characteristics $C_{4}^{ \pm}: x-t=\sigma^{ \pm}$(see Fig. 2). The crossing points of $C_{4}^{+}$ with $C_{3}, C_{2}, C_{1}$ are denoted by $A_{1}, A_{2}, A_{3}$ and their ordinates are $a_{1}, a_{2}, a_{3}$, respectively. The crossing points $B_{1}, B_{2}, B_{3}$ and their ordinates $b_{1}, b_{2}, b_{3}$ are introduced in a similar way as above (see Fig. 2). The smooth functions $a_{3}(\sigma)>0, b_{3}(\sigma)<0$ satisfy the equation $\alpha+\sigma=\alpha^{p}, \sigma=x-t, \alpha(0)=0$, the functions $a_{1}(\sigma)>0, b_{1}(\sigma)<0$ satisfy the equation $\beta(\sigma)+\sigma=-\beta^{p}(\sigma), \beta(0)=0$ and $a_{2}, b_{2}$ are the solutions of $\gamma(\sigma)+\sigma=0$. The values of $w$ under the parabolas $C_{1}, C_{3}$ are denoted by $w_{1}(x, t)$, the values of $w$ inside $C_{1}$ are denoted by $w_{2}(x, t)$, the values of $w$ in the curvilinear angle between $C_{2}^{+}, C_{1}^{+}$are denoted by $w_{3}(x, t), C_{1}^{+}=C_{1} \cap\{x>0\}$. The definition of $w_{4}$ is obvious. One can easily see that

$$
\begin{gathered}
w_{1}=\int_{-T}^{t}\left(1+v_{1}\right) d \tau, \quad t<\tau_{1} \\
w_{2}=w_{1}+\int_{\tau_{1}}^{t}\left(c_{2}-2 \tau^{p}\right) d \tau+\int_{\tau_{1}}^{t}\left(v_{2}-v_{1}\right) d \tau, \quad \tau_{1} \leq t<\tau_{2}
\end{gathered}
$$

and we are integrating along the curve $\left(c_{2}-\tau^{p}, \tau\right)$,

$w_{3}=\int_{-T}^{t}\left(1+v_{1}\right) d \tau+\int_{\tau_{1}}^{\tau_{2}}\left(c_{2}-2 \tau^{p}\right) d \tau+\int_{\tau_{1}}^{\tau_{2}}\left(v_{2}-v_{1}\right) d \tau+\int_{\tau_{2}}^{t} \frac{2 p}{p+1}\left(c_{2}-\tau^{p}\right)^{1+1 / p} d \tau$,

$\tau_{2} \leq t<\tau_{3}$. A similar expression can be found for $w_{4}$. The previous integrals can be investigated via the standard change $\tau=c_{2}^{1 / p} s$. Obviously,

$$
z^{+}=\int_{-T}^{a_{1}}(\cdot) d \tau+\int_{a_{1}}^{a_{2}}(\cdot) d \tau+\int_{a_{2}}^{a_{3}}(\cdot) d \tau+\int_{a_{3}}^{t}(\cdot) d \tau
$$


where the underintegral function is $\alpha u v+\beta u w+\gamma v w$ and we are integrating along the straight line $(\tau+\sigma, \tau), \sigma=x-t$. Similar decomposition holds for $z^{-}$. As in (1), case d), the term $\alpha u v$ leads to a jump discontinuity of sharp order $2 p$ along $C_{4}$ and inside $C_{1}$. The singularities could appear because of the presence of "asymmetric terms" in the expressions for $z^{ \pm}$. These terms are of the type

$$
\int_{a_{1}}^{a_{2}} c_{2}^{1+1 / p} d \tau, \int_{a_{1}}^{a_{2}} c_{2}^{1+2 / p} d \tau, \int_{a_{2}}^{a_{3}} c_{2}^{1+1 / p} d \tau, \ldots, c_{2}=\left(\tau+\sigma+\tau^{p}\right)^{1+1 / p}
$$

In order to find out the leading singularity, say in the third integral in (7), we make the standard change $\tau+\sigma=s^{p}$, having in mind that $\sigma<0$. Applying the binomial power series we come to the conclusion that the integral under consideration possesses the following leading term: const. $\sigma^{p+1} a_{3}^{p}=o\left(\sigma^{2 p+1}\right), \sigma \rightarrow 0^{-}$. This way we come to the following proposition.

Proposition 2. Consider the semilinear weakly hyperbolic system (6) with Cauchy data $u_{0}$ having finite jump of order $k=0$ at some point $x_{0}$ and $v_{0}=w_{0}=z_{0}=0$. Then in general the component $z$ does not have finite jump of exact order $(2 k+3) p$ along the characteristic $C_{4}$ located inside the parabola $C_{1}$.

In our case the jump discontinuity is of order $(k+2) p<(2 k+3) p$. This is a new effect, of course.

2.3. We shall deal now with the following weakly hyperbolic system

$$
\left\{\begin{array}{l}
X u=\left(\partial_{t}+q t^{q-1} \partial_{x}\right) u=0 \\
\partial_{t} v=u \\
Y w=\left(\partial_{t}-p t^{p-1} \partial_{x}\right) w=u+v \\
D z=\left(\partial_{t}+\partial_{x}\right) z=\alpha u v+\beta u w+\gamma v w \\
\quad \alpha, \beta, \gamma=\text { const., } p, q-\text { even, } q>p \geq 2,
\end{array}\right.
$$

with Cauchy data $\left.u\right|_{t=-T}=u_{0}(x), T>0, v_{0}=w_{0}=z_{0} \equiv 0$, where $u_{0}$ is defined as in case d) for system (1).

The characteristics of the vector fields $X, \partial_{t}, Y, D$ passing through the point $(\bar{x}, \bar{t})$ are given respectively by the equations $x=t^{q}+c_{1}, c_{1}=\bar{x}-\bar{t}^{q}, x=\bar{x}, x=-t^{p}+c_{2}$, $c_{2}=\bar{x}+\bar{t}^{p}, x-t=\bar{x}-\bar{t}$. Put $C_{1}: x=t^{q}, C_{2}: x=0, C_{3}: x=-t^{p}, \bar{C}_{3}: x=-t^{p}+c_{2}$, $C_{4}: x=t$. Evidently, $C_{1}, C_{2}, C_{3}$ are tangential each to other characteristics at the origin and $C_{4}$ is transversal to them at $O$. The main problem we are interested in is whether the new-born singularity at $O$ of the component $z$ will have a finite jump along $C_{4}$ and inside $C_{1}$ depending on both integers $p$ and $q$. As our considerations are similar to that realized for the system (6) we shall omit some details. One can easily see that $u=u_{1}=1$ outside the parabola $C_{1}$ and $u=u_{2}=1+x-t^{q}$ inside it. By $v_{i}, i=1,2,3$, we denote the values of $v$ in the subdomains $\{x<0\} \cup\left\{0<x<t^{q}, t<0\right\},\left\{t^{q}<x\right\}$ and the curvilinear angle between $C_{1}^{+}, C_{2}^{+}$. Then

$$
v_{1}=t+T, \quad v_{2}=v_{1}+x t-\frac{t^{q+1}}{q+1}+\frac{q}{q+1} x^{1+1 / q}, \quad v_{3}=v_{1}+\frac{2 q}{q+1} x^{1+1 / q} .
$$

Put $c_{2}=x+t^{p}$. Obviously, $w(x, t)=\int_{-T}^{t}(u+v)\left(c_{2}-\tau^{p}, \tau\right) d \tau$. 
The crossing points of $\bar{C}_{3}$ with $C_{1}$ have ordinates $\tau_{2}=-\tau_{1}>0$ which satisfy the equation $\tau^{p}+\tau^{q}=c_{2}>0$. Let $\xi=c_{2}^{1 / p}$ and $\rho(\xi)=\tau(\xi), \rho(\xi)=\xi(1+\pi)$. Therefore, $(1+\pi)^{p}+\xi^{q-p}(1+\pi)-1=0$. Applying the implicit function theorem we conclude that in a neighbourhood of the point $\xi=0, \pi=0$ there exists a unique function $\pi(\xi) \in C^{\infty}, \pi(0)=0$, satisfying $\rho^{p}(\xi)+\rho^{q}(\xi)=\xi$. More detailed analysis shows that $\pi(\xi)=-\frac{1}{p} \xi^{q-p}(1+O(\xi)), \xi \rightarrow 0$. So $\tau_{2}=c_{2}^{1 / p}\left(1+\pi\left(c_{2}^{1 / p}\right)\right), \pi(0)=0$. The ordinate of the crossing point of $C_{2}^{+}$and $\bar{C}_{3}$ is $\tau_{3}=c_{2}^{1 / p}$. The crossing points of $C_{4}^{+}$with $C_{3}, C_{2}, C_{1}$ are denoted by $A_{1}, A_{2}, A_{3}$ and their ordinates are $a_{1}, a_{2}, a_{3}$, respectively. The crossing points $B_{1}, B_{2}, B_{3}$ and their ordinates $b_{1}, b_{2}, b_{3}$ are defined similarly. Put $\sigma=x-t$. Then $\left(a_{1}, b_{1}\right),\left(a_{2}, b_{2}\right),\left(a_{3}, b_{3}\right)$ satisfy the equations $\tau+\tau^{p}+\sigma=0, \tau+\sigma=0, \tau^{q}=\tau+\sigma$, respectively. An application of the inverse function theorem to $\tau^{q}-\tau=\sigma$ near $\tau=0$ shows that one can find a unique $C^{\infty}$ smooth inverse function $\tau=\tau(\sigma),|\sigma| \ll 1, \tau(0)=0$ and $\tau(\sigma)=-\sigma+\sigma^{q}+O\left(\sigma^{q+1}\right), \sigma \rightarrow 0$. So $a_{3}, b_{3}=-\sigma_{ \pm}+\sigma^{q}+O\left(\sigma_{ \pm}^{q+1}\right)$. The values $w_{i}$, $1 \leq i \leq 4$ of $w$ are found in a similar way as in the case of system (6) in the intervals $t<\tau_{1}, \tau_{1} \leq t<\tau_{2}, \tau_{2} \leq t<\tau_{3}, t \geq \tau_{3}$. We have $w_{1} \in C^{\infty}$. Then for $t \in\left(\tau_{1}, \tau_{2}\right]$,

$$
w_{2}=w_{1}+\int_{\tau_{1}}^{t}\left(c_{2}-\tau^{p}-\tau^{q}\right) d \tau+\int_{\tau_{1}}^{t}\left[\left(c_{2}-\tau^{p}\right) \tau-\frac{\tau^{q+1}}{q+1}+\frac{q}{q+1}\left(c_{2}-\tau^{p}\right)^{1+1 / q}\right] d \tau .
$$

Making the change $\tau=c_{2}^{1 / p} s$ we get

$$
\int_{\tau_{1}}^{t}\left(c_{2}-\tau^{p}\right)^{1+1 / q} d \tau=\int_{-\left(1+\pi\left(c_{2}^{1 / p}\right)\right)}^{t / c_{2}^{1 / p}}\left(1-s^{p}\right)^{1+1 / q} d s c_{2}^{1+1 / p+1 / q} .
$$

As we are inside the parabola $C_{1}$ we have $x>0$ and therefore the previous integral is $C^{\infty}$ smooth there. Having in mind the formulas giving the values of $\tau_{i}, 1 \leq i \leq 3$, we obtain

$$
\begin{gathered}
w_{2}=w_{1}+\frac{p}{p+1} c_{2}^{1+1 / p}\left(1+O\left(c_{2}^{1 / q}\right)+O\left(c_{2}^{(q-p) / p}\right)\right), \quad c_{2} \rightarrow 0 \\
w_{1} \in C^{\infty}, \quad w_{1}=\int_{-T}^{t}\left(1+v_{1}\right) d \tau
\end{gathered}
$$

Similar considerations show that

$$
\begin{array}{ll}
w_{3}=w_{1}+\frac{p}{p+1} c_{2}^{1+1 / p}\left(1+O\left(c_{2}^{1 / q}\right)+O\left(c_{2}^{(q-p) / p}\right)\right), & c_{2} \rightarrow 0, \\
w_{4}=w_{1}+\frac{2 p}{p+1} c_{2}^{1+1 / p}\left(1+O\left(c_{2}^{1 / q}\right)+O\left(c_{2}^{(q-p) / p}\right)\right), \quad c_{2} \rightarrow 0 .
\end{array}
$$

The expressions for $z^{+}$and $z^{-}$are the same as in the previous case (6) and we are integrating along the straight lines $\left(\tau+\sigma_{+}, \tau\right),\left(\tau+\sigma_{-}, \tau\right), \sigma_{ \pm}=x-t$. The term $\alpha u v$, $\alpha \neq 0$, leads to a jump discontinuity of sharp order $2 q$ along the part of the characteristic $C_{4}$ located inside $C_{1}$. As was mentioned above, the jump discontinuities could appear because of the presence of several "asymmetric terms" in the expressions for $z^{ \pm}$. Let us concentrate now on the right-hand side $\beta u v, \beta \neq 0$. The leading "asymmetric terms" are: $\int_{a_{1}}^{a_{2}} w_{4} d \tau, \int_{a_{2}}^{a_{3}} w_{3} d \tau, \int_{b_{1}}^{b_{2}} w_{1} d \tau, \int_{b_{2}}^{b_{3}} w_{1} d \tau$. To fix the ideas we shall deal with the first two integrals only. One can easily guess that the leading singularities in that situation 
(i.e. the terms containing the lowest powers of $\sigma$ ) are

$$
\mathrm{I}=\int_{a_{1}}^{a_{2}} c_{2}^{1+1 / p} d \tau, \quad \mathrm{II}=\int_{a_{2}}^{a_{3}} c_{2}^{1+1 / p} d \tau
$$

and the integration in the previous integrals is along the line $(\tau+\sigma, \tau)$.

There are no difficulties to verify the existence of a smooth function $a(\sigma), \sigma \ll 1$, $0<a(\sigma)<-\sigma, \sigma>0$ with the properties $\tau \in[a(\sigma),+\infty) \Rightarrow \tau+\sigma+\tau^{p}=h(\tau) \geq 0$, $h(a)=0, h^{\prime}(\tau)>0$. Then $h^{-1}$ exists on the interval $[0, \infty)$ and is strictly monotone there. Following this way we conclude that the smooth function $s(\tau)=\left(\tau+\sigma+\tau^{p}\right)^{1 / p}$ is well defined and invertible on $[a(\sigma), \infty)$. Moreover, the inverse function

$$
\tau(s)=a(\sigma)+\frac{s^{p}}{1+p a^{p-1}(\sigma)}+O\left(s^{2 p}\right), \quad s \rightarrow 0, s \geq 0 .
$$

Now we are able to make the following change in I: $s^{p}=\tau+\sigma+\tau^{p}, \tau \geq a(\sigma) \geq 0$. Then $\tau=a_{1} \Rightarrow s_{1}=0, \tau=a_{2} \Rightarrow s_{2}=a_{2}>0$. Thus

$$
\mathrm{I}=p \int_{0}^{a_{2}} \frac{s^{2 p}}{1+p \tau^{p-1}(s)} d s=\text { const. } a_{2}^{2 p+1}\left(1+O\left(a_{2}^{2 p+2}\right)\right), \quad a_{2} \rightarrow 0 ; \quad \text { const. } \neq 0 .
$$

Consider now the function $s^{p}+s^{q}=h_{1}(s), s \geq 0$. Certainly, it is strictly monotonically increasing and $s=h^{-1}(w)=w^{1 / p}+O\left(w^{2 / p}\right), w \rightarrow+0$. Let us make now in the second integral II the change $s^{p}+s^{q}=\tau+\sigma+\tau^{p}, \tau \geq a(\sigma)$ (i.e. $s=h_{1}^{-1}\left(\tau+\sigma+\tau^{p}\right), \tau \geq a(\sigma)$, $\sigma$ - parameter). Then $\tau=a_{2} \Rightarrow s_{1}=h_{1}^{-1}\left(a_{2}^{p}\right)=a_{2}\left(1+O\left(a_{2}\right)\right)$.

Thus

$$
\mathrm{II}=\int_{h_{1}^{-1}\left(a_{2}^{p}\right)}^{a_{3}} s^{2 p}\left(1+s^{q-p}\right)^{1+1 / p} \frac{\left(p+q s^{q-p}\right)}{1+p \tau^{p-1}(s)} d s=O\left(a_{2}^{2 p+2}\right), \quad a_{2} \rightarrow 0 .
$$

Hence, $\mathrm{I}=$ const. $\sigma^{2 p+1}\left(1+O\left(\sigma^{2 p+2}\right)\right)$, II $=O\left(\sigma^{2 p+2}\right)$; const. $\neq 0$.

Proposition 3. Consider the semilinear hyperbolic system (8) with Cauchy data $u_{0}$ having a finite jump of order $k=0$ at some point $x_{0}$ and $v_{0}=w_{0}=z_{0}=0$. Then in general the component $z$ does not have a finite jump of exact order $(2 k+3) p$ along the characteristic $C_{4}$ located inside the parabola $C_{1}$. In our case the jump discontinuity is of order $(k+2) p, k=0$ and therefore it does not depend on $q$.

In other words we cannot expect interaction between the orders of tangency $p$, respectively $q$, of the characteristics $C_{1}, C_{2}$ and $C_{2}, C_{3}$ at the origin in determining the strength of the new-born jump discontinuity of the component $z$ along the semicharacteristic $C_{4} \cap\{x>0\}$.

Short summary of results in the communication. Two, respectively three, characteristics of the semilinear systems under consideration are assumed to be mutually tangential of order $p \geq 2$ at 0 and the Cauchy data are continuous with a jump discontinuity of the first derivative at a given point $x_{0}$. Then a newly created singularity appears at 0 and propagates along a transversal outgoing characteristic. The sharp order of that jump discontinuity is $2 p$ instead of the expected order $3 p$ according to [7], [10]. 
Acknowledgements. The first author is grateful to the organizers of the Conference on Evolution Equations held in Warsaw, Banach Center, in July 2001 for the invitation and the support.

\section{References}

[1] M. BEALs, Self-spreading and strength of singularities for solutions to semi-linear wave equations, Ann. of Math. (2) 118 (1983), 187-214.

[2] J.-M. Bony, Propagation des singularités pour les équations aux dérivées partielles non linéaires, Séminaire Goulaouic-Schwartz (1979-1980), Exp. No. 22, École Polytechnique, Palaiseau, 1980.

[3] J.-M. Bony, Analyse microlocale des équations aux dérivées partielles non linéaires, in: Microlocal Analysis and Applications (Montecatini Terme, 1989), L. Cattabriga and L. Rodino (eds.), Lecture Notes in Math. 1495, Springer, Berlin, 1991, 1-45.

[4] J.-Y. Chemin, Interaction des trois ondes dans les équations sémilinéaires strictement hyperboliques d'ordre 2, Comm. Partial Differential Equations 12 (1987), 1203-1225.

[5] T. Gramchev, Nonlinear maps in spaces of distributions, Math. Z. 209 (1992), 101-114.

[6] L. Hörmander, Lectures on Nonlinear Hyperbolic Differential Equations, Math. Appl. 26, Springer, Berlin, 1997.

[7] I. Iordanov, Anomalous singularities of semilinear nonstrictly hyperbolic system, J. Math. Pures Appl. (9) 70 (1991), 393-426.

[8] F. John, Nonlinear Wave Equations, Formation of Singularities, Univ. Lecture Ser., Amer. Math. Soc., Providence, 1990.

[9] R. Melrose and N. RitTeR, Interaction of nonlinear progressing waves for semilinear equations, Ann. of Math. (2) 121 (1985), 187-213.

[10] L. Micheli, Propagation of singularities for nonstrictly hyperbolic semilinear systems in one space dimension, Trans. Amer. Math. Soc. 291 (1985), 451-485.

[11] J. RAuch and M. REed, Propagation of singularities for semilinear hyperbolic equations in one space variable, Ann. of Math. (2) 111 (1980), 531-552.

[12] J. RAUCH and M. REED, Propagation of singularities in nonstrictly hyperbolic semilinear systems: examples, Comm. Pure Appl. Math. 35 (1982), 555-565. 